\title{
Poster: Apps Can Quickly Destroy Your Mobile's Flash - Why They Don't, and How to Keep It That Way
}

\author{
Tao Zhang \\ The University of North Carolina at Chapel Hill \\ zhtao@cs.unc.edu \\ Donald E. Porter \\ The University of North Carolina at Chapel Hill \\ porter@cs.unc.edu
}

\section{CCS CONCEPTS}

- Information systems $\rightarrow$ Flash memory; • Security and privacy $\rightarrow$ Mobile platform security.

\section{ACM Reference Format:}

Tao Zhang, Aviad Zuck, Donald E. Porter, and Dan Tsafrir. 2019. Poster: Apps Can Quickly Destroy Your Mobile's Flash - Why They Don't, and How to Keep It That Way. In The 17th Annual International Conference on Mobile Systems, Applications, and Services (MobiSys '19), June 17-21, 2019, Seoul, Republic of Korea. ACM, New York, NY, USA, 2 pages. https: //doi.org/10.1145/3307334.3328729

\section{INTRODUCTION}

Smartphones typically include flash-based storage, because flash offers benefits such as fast random access, shock resistance, high density, and decreasing costs. A main drawback, however, is that flash cells can tolerate only a limited number of writes before becoming unusable.

Although flash cells wear out, a typical SSD has enough cells and sufficiently sophisticated firmware that its lifetime generally exceeds the expected lifetime of its host system. Even under heavy use, SSDs last for years. On a smartphone, in contrast, the hardware is more limited and we show that, under heavy use, one can easily, and more quickly, wear out smartphone flash storage. Consequently, a simple, unprivileged, malicious application can render a smartphone unbootable ("bricked") in a few weeks with no warning signs to the user. This bleak result becomes more worrisome when considering the fact that smartphone users generally believe it is safe to try out new applications.

To combat this problem, we study the I/O behavior of a wide range of Android applications. We find that high-volume write bursts exist, yet none of the applications we checked sustains an average write rate that is high enough to damage the device, under reasonable usage assumptions backed by the literature. We therefore propose a rate-limiting algorithm for write activity that (1) prevents such attacks; (2) accommodates "normal" bursts; and

Permission to make digital or hard copies of part or all of this work for personal or classroom use is granted without fee provided that copies are not made or distributed for profit or commercial advantage and that copies bear this notice and the full citation on the first page. Copyrights for third-party components of this work must be honored.

For all other uses, contact the owner/author(s).

MobiSys '19, June 17-21, 2019, Seoul, Republic of Korea

(C) 2019 Copyright held by the owner/author(s).

ACM ISBN 978-1-4503-6661-8/19/06

https://doi.org/10.1145/3307334.3328729

\author{
Aviad Zuck \\ Technion - Israel Institute of Technology \\ aviadzuc@cs.technion.ac.il \\ Dan Tsafrir \\ Technion - Israel Institute of Technology \& \\ VMware Research \\ dan@cs.technion.ac.il
}

(3) ensures that the smartphone drive lifetime is longer than a pre-configured lower bound (i.e., its warranty). In terms of user experience, our design only requires that, in the worst case of an app that issues continuous, unsustainable, and unusual writes, the user decides whether to shorten the phone's life or rate-limit the problematic app.

The key contributions of this work include: (1) an empirical evaluation of the lifespan of flash storage on a range of mobile phones; (2) a characterization of the write I/O behavior of a wide range of benign smartphone apps, with a particular focus on write-intensive apps; (3) an empirical explanation of why benign apps do not wear out smartphone flash in practice; (4) a defense against dangerous I/O behaviors and a wear-out attack, using unmodified, commodity hardware.

\section{BACKGROUND}

Flash storage is composed of large arrays of physical wordlines, which are serially connected floating-gate cells [1]. Data is read and written at page units, corresponding in size to physical wordlinestypically 4-16 KiB. Data cannot be updated in-place, instead an erase operation is needed to get the cells ready for new writes. Erasure happens at a larger granularity, called a block, typically 256-4096 KiB in size. In order to ensure that blocks are erased and available to accept new writes without losing live data, most SSDs have a management firmware, called a flash translation layer (FTL), which reclaims and internally migrates live data to different physical blocks.

Flash blocks are limited in the number of Program/Erase (P/E) cycles they can endure. Although various mechanisms can improve the lifetime of flash storage, recent, popular techniques that optimize capacity tend to sacrifice lifespan; many modern flash chips endure 1-3K only $\mathrm{P} / \mathrm{E}$ cycles [2].

\section{WEAR-OUT DEVICES}

We measure the performance characteristics of a collection of mobile flash storage devices (Table 1). We then measure how fast these flash storage wear-out under different I/O patterns. Our full paper [3] and poster present results indicating that these devices can be worn out in weeks.

We design a proof-of-concept wear-out attack targeting real-life mobile systems using only a simple, virtually permission-less app, called WAPP. WAPP can be installed and run on an benign Android system, requiring only the ability to write data into either a private 


\begin{tabular}{|c|c|}
\hline Device (Storage info) & OS \\
\hline $\begin{array}{l}\text { eMMC 8GB } \\
\text { (THGBMBG6D1KBAIL eMMC 5.0) }\end{array}$ & \multirow{3}{*}{$\begin{array}{l}\text { Ubuntu } 16.04 \\
\text { (kernel } 3.14 .79\end{array}$} \\
\hline eMMC 16GB (iNAND 7030 eMMC 5.0) & \\
\hline$\mu \mathrm{SD} 16 \mathrm{~GB}$ (Kingston SDC4/16GB) & \\
\hline $\begin{array}{l}\text { Samsung S9 64GB } \\
\text { (Toshiba THGAF8G9T43BAIR UFS 2.1) }\end{array}$ & Android 9 \\
\hline $\begin{array}{l}\text { Samsung S6 32GB } \\
\text { (Samsung KLUBG4G1BE-E0B1 UFS 2.0) }\end{array}$ & Android 6.0.1 \\
\hline Moto E 8GB (Samsung QN1SMB eMMC 4.5) & Android 5.1 \\
\hline BLU 512MB (K524G2GACH-B050 NAND Flash) & \multirow{2}{*}{ Android 4.4} \\
\hline BLU 4GB (TYC0FH121638RA eMMC) & \\
\hline
\end{tabular}

Table 1: Evaluated mobile storage devices.

or shared storage space, which is granted by default to most apps. WAPP continuously and sequentially rewrites $100 \mathrm{MiB}$ files in the app's private storage space. To avoid raising attention from the system and user, WAPP only launches the attack stealthily in the background, when the phone is charging and the screen is off.

Figure 1 shows the time for WAPP to increment the wear indicator by one; the indicator goes up to 11 , at which point the storage is considered unreliable. In general, this is measured in hours, and a typical phone we measured will reach 11 within weeks under this attack, even on a new phone with a 1-2 year warranty. Additional details of these experiments are available in our full paper [3].

\section{MOBILE APP I/O CHARACTERIZATION}

A goal of this work is to defend against premature wear-out and wear-out attacks without harming normal app behavior or user experience. Thus, an important question to ask is, what level of write bandwidth is typically used by current mobile apps? With this information, we can assess the potential to destroy the device in practice, as well as whether one can differentiate benign and dangerous write behavior.

We study the I/O characteristics of a collection of popular apps, including 27 pre-loaded apps and the top 150 free apps from Google Play store. The results show that a) some write-heavy apps can utilize $\sim 5 \mathrm{MiB} / \mathrm{s}$ average throughput (with bursts at over $100 \mathrm{MiB} / \mathrm{s}$ ), which is higher than the safe lifetime average throughput $1.46 \mathrm{MiB} / \mathrm{s}$, but these bursts are not sustained for long periods; b) most apps cause little to no background I/O. However, even for write-heavy apps, usage scenarios that require nonstop operation are unlikely. Under reasonable usage assumptions, the vast majority of apps do not display I/O behavior which causes significant wear on the device.

\section{MANAGING WEAR}

Finally, we contribute a rate-limiting solution to protect against wear-out attacks. We model our device as having a total warrantied lifetime write $W$, with a target lifetime $L$ (e.g., for the Samsung S6 32GB phone, $W=88 \mathrm{TiB}, L=2$ years). We allocate $S=W / 2$ as slack write capacity, in order to accommodate bursts of $\mathrm{I} / \mathrm{O}$, which is common in most apps. To prevent a potential denial-ofservice attack that could rapidly exhaust $S$ and rate limit all other apps, $S$ is allocated on a daily basis as a quota for all apps, $S_{d a y}$.

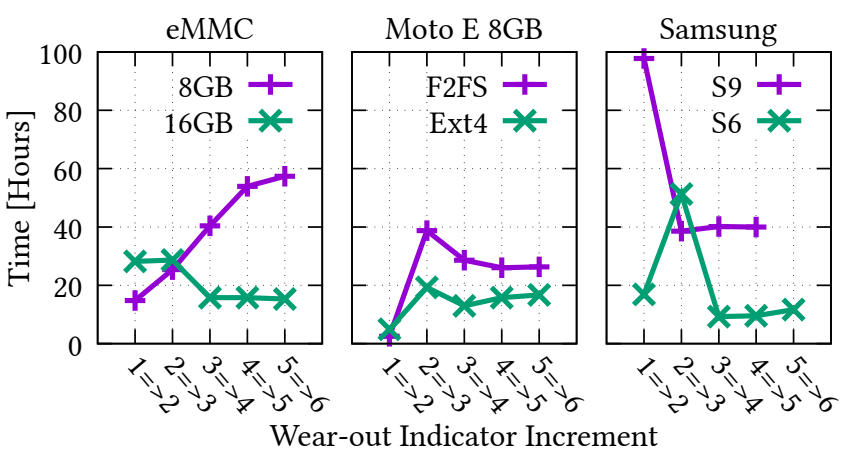

Figure 1: Time to increment wear-out indicators ${ }^{1}$ on two smartphone models and two external eMMC chips.

Because our characterization study indicates that apps running in the background tend to issue fewer writes, we limit $S_{d a y}$ into an hourly slack budget for background apps $S_{b g}$.

We evaluate our solution on the Samsung S6 with Android 6.0.1 and Linux kernel 3.10.101. The implementation includes an extension to Linux kernel which tracks application I/O behavior, as well as a policy executor that can dynamically apply a configurable write-limiting policy. Evaluation results show that our solution can effectively detect and throttle the WAPP attack, while inducing negligible interference on benign apps.

\section{CONCLUSION}

Mobile OSes assume, often incorrectly, that it is safe to allow apps to issue unconstrained writes to the device. Our results show that the ratio of bandwidth to capacity in the underlying device is often dangerously skewed, and the firmware is likely less able than an SSD to tolerate heavy writes. We demonstrate how this can be corrected at the system software level with a carefully designed rate-limiting algorithm and configuration, without disrupting normal behavior.

This poster focuses on smartphones, but we believe the same issues apply to any small, flash-based devices on which third-party software can be loaded, potentially including critical infrastructure or internet-connected medical devices. The continuing proliferation of IoT and embedded devices to everyday life also presents new opportunities to attack high-bandwidth, mobile, flash-based, storage devices with relatively small capacity that can be maliciously worn-out.

\section{REFERENCES}

[1] Eran Gal and Sivan Toledo. 2005. Algorithms and Data Structures for Flash Memories. Computing Surveys 37, 2 (June 2005), 138-163. https://doi.org/10.1145/ 1089733.1089735

[2] Bianca Schroeder, Raghav Lagisetty, and Arif Merchant. 2016. Flash Reliability in Production: The Expected and the Unexpected. In 14th USENIX Conference on File and Storage Technologies (FAST). USENIX Association, 67-80. http://usenix.org/ conference/fast16/technical-sessions/presentation/schroeder

[3] Tao Zhang, Aviad Zuck, Donald E. Porter, and Dan Tsafrir. 2019. Apps Can Quickly Destroy Your Mobile's Flash: Why They Don't, and How to Keep It That Way. In ACM International Conference on Mobile Systems, Applications, and Services (MobiSys).

${ }^{1}$ Indicator value of $n$ means the storage chip's consumed lifetime is between $(n-1) *$ $10 \%$ and $n * 10 \%$. 